\title{
HETEROGENEITY IN PREFERENCES TOWARDS COMPLEXITY
}

\author{
Peter G. MOFFATT, ${ }^{*}$ Stefania SITZIA, ${ }^{* *}$ Daniel John ZIZZO***
}

\section{October 2015}

\begin{abstract}
We analyze lottery-choice data in a way that separately estimates the effects of risk aversion and complexity aversion. Complexity is represented by the number of different outcomes in the lottery. A finite mixture random effects model is estimated which assumes that a proportion of the population are complexity-neutral. We find that around $33 \%$ of the population are complexity-neutral, around 50\% complexity-averse, and the remaining 17\% are complexityloving. Subjects who do react to complexity appear to have a bias towards complexity aversion at the start of the experiment, but complexity aversion reduces with experience, to the extent that the average subject is (almost) complexity-neutral by the end of the experiment. Complexity aversion is found to increase with age and to be higher for non-UK students than for UK students. We also find some evidence that, when evaluating complex lotteries, subjects perceive probabilities in accordance with Prospective Reference Theory.
\end{abstract}

Keywords: complexity aversion, complexity preferences, risk preferences, mixture models, learning.

JEL Classification Codes: C91, D03, D81.

* School of Economics, Norwich Research Park, University of East Anglia, Norwich NR4 7TJ, United Kingdom. Email address: p.moffatt@uea.ac.uk

** School of Economics and CBESS, Norwich Research Park, University of East Anglia, Norwich NR4 7TJ, United Kingdom. Email address: s.sitzia@uea.ac.uk

*** Newcastle University Business School and BENC, Newcastle University, 5 Barrack Road, Newcastle upon Tyne NE1 2JE, United Kingdom. Email address: daniel.zizzo@ncl.ac.uk

Funding from the University of East Anglia, advice from Bob Sugden and from participants at the Asia Pacific meeting of the Economic Science Association in Auckland in February 2014, and research assistance from Axel Sonntag, are gratefully acknowledged. The usual disclaimer applies. The experimental instructions can be found in the appendix. 
That complexity matters in economic decisions and other realms of human life is neither surprising nor new. Previous studies have mainly focused on understanding how complexity affects the accuracy of choices (Bruce and Johnson 1996), how this may be exploited by firms (Ellison and Ellison, 2004), how complexity leads to a lower evaluation of lotteries (Mador et al., 2000), and how complexity avoidance can lead to suboptimal portfolio selection (Sonsino et al. 2002).

Theoretical research on preferences towards complexity appears predisposed to an assumption of complexity aversion. Stodder (1997) uses complexity aversion to explain the Allais paradox. Gale and Sabourian (2005) show that complexity aversion can lead to a preference for competitive over non-competitive outcomes in market games. In order for these theoretical models to be at least plausible, it is important to establish how widespread the phenomenon of complexity aversion is in the population.

This is the primary aim of the present paper, in which experimental data is used to estimate the distribution of preferences towards complexity in the population. We do this in conjunction with the estimation of preferences towards risk. Thus we pursue one of the secondary goals of the paper: to assess the relationship, if any, between preferences towards complexity and preferences towards risk.

Some previous empirical work on complexity preferences appears to favour complexity aversion. Huck and Weizsäcker (1999) and Sonsino et al. (2002) both find evidence of complexity aversion in lottery choice. Bruce and Johnson (1997), however, find no evidence of complexity aversion in horse-track betting decisions. Some evidence of complexity aversion has been found in choices over products (Rouse, 2008) and choices over energy tariffs (Garrod et al., 2008; Sitzia et al., 2012). Sitzia and Zizzo (2011) find experimental evidence that consumers are exploitable (i.e. buy greater quantities at higher prices) when products are complex.

Alongside this evidence of complexity aversion, some counter-evidence exists which raises the possibility that a proportion of the population is complexity-neutral or even complexityloving. Complexity neutrality is naturally implied by standard theories of decision making under risk, including expected utility theory. Complexity lovingness is a potential explanation for the "event splitting" effect. The event splitting effect, observed by Starmer and Sugden (1993), Humphrey $(1995,2001)$ and Weber (2007), is the phenomenon of a lottery becoming more appealing to subjects when one of the outcomes is presented as two different identical outcomes. Such evidence leads us to conjecture that more complex lotteries might be preferred because they are perceived as offering "more ways to win" (Weber, 2007).

To anticipate our key finding, we are able to estimate a posterior distribution of complexity preferences in our experimental sample while controlling for risk preferences, with some $50 \%$ of the sample being complexity-averse, $33 \%$ being complexity-neutral and $17 \%$ complexityloving, but also with complexity-averse subjects tending to converge towards complexity neutrality with experience. We find that our preference distribution helps explain choice switches in our experiment.

The paper is organized as follows. Section 1 describes Sonsino et al.'s (2002) experiment. The reason why we focus on that experiment is because our experimental design extends theirs. Sections 2 and 3 describe the experimental method and present the experimental design. Section 4 presents exploratory data analysis, including non-parametric tests for complexity aversion. Section 5 briefly outlines the econometric model. Section 6 presents and discusses the estimation results. Section 7 concludes. 


\section{Sonsino et al.'s (2002) experiment}

Sonsino et al. (2002) designed an experiment to study the effects of complexity on lottery choices, with complexity being measured in terms of the number of outcomes in the lottery. Lotteries all have the same expected value (107 experimental points) but different complexity levels.

Our own design (see Section 3) directly reproduces two of the choice tasks in Sonsino et al.'s design (2002, pp. 950-952; Problems 11 and 12). These two tasks are presented in Table 1 below. The first task was to choose between a sure win (labelled SW in our design) and a "simple" lottery with three outcomes (S3 in our design); the second was to choose between that same "simple" lottery (S3) and a "complex" lottery with 6 outcomes (C3 in our design). The two tasks are presented in Table 1.

\section{(Insert Table 1 about here)}

These two tasks provide a useful example for drawing attention to two important and closely related features of the design. Firstly, Sonsino et al. (2002) have used a particular procedure to derive the "complex" lottery C3 from the "simple" lottery S3. The procedure is best described by imagining that S3 is played twice in succession, with the total outcome from both plays then being divided by 2 . This procedure inevitably results in a more complex lottery; however, it is also the case that the complex lottery is less risky than the simple lottery, in the sense of having a smaller variance. In our own design (see next section), this procedure is generalised, and used to derive "very complex" lotteries (with 27 outcomes) from "complex" lotteries.

Secondly, the presence in the design of complex lotteries that are less risky than simple lotteries is essential for separating the effect of complexity aversion from that of risk aversion. Consider the two tasks presented in Table 1. If a subject is both risk-averse and EUT maximizing, she should prefer SW over S3, and C3 over S3. If she is risk seeking and EUT maximizing, she should prefer S3 in both tasks. However, a subject who chooses SW in the first task, and S3 in the second, is signalling that she is complexity-averse, since she is apparently being put off by the complexity of $\mathrm{C} 3$ in the second task.

Of course, such "preference reversals" may simply be a consequence of noisiness in choice. In order to detect the presence of complexity aversion, it is necessary also to consider the number of reversals in the opposite direction (i.e. from S3 in task 1 to $\mathrm{C} 3$ in task 2). If the number of subjects choosing SW and S3 is significantly greater than the number choosing S3 and C3, this may be interpreted as evidence of complexity aversion in the population. A nonparametric test appropriate for making this comparison formally is the McNemar test (Siegel and Castellan, 1988). Sonsino et al. find that, of their 120 subjects, 36 switched from SW to S3, while only 19 switched from S3 to C3. The McNemar test statistic $\left(\chi^{2}(1)\right)$ obtained from these numbers is 6.90 , and the $p$-value is 0.0086 , indicating strong evidence of complexity aversion on the basis of these two sets of choices. The McNemar test will be applied more extensively to our own data in Section 4.

\section{Experiment}

The experiment was run at the University of East Anglia in February $2013 .{ }^{1}$

\footnotetext{
${ }^{1}$ The experiment was programmed and conducted with the experiment software z-Tree (Fischbacher, 2007).
} 
A sample of 80 subjects took part in the experiment. After their arrival, subjects were asked to read the instructions and then to complete a questionnaire to check whether they understood the type of task encountered in the experiment. ${ }^{2}$ Subjects were then given an opportunity to ask questions of clarification. Then the experiment commenced.

The experiment consisted of two phases. In each phase, subjects faced 27 tasks in which they were asked to choose between two lotteries with the same expected value of 107 experimental points $(10$ points $=£ 1)$, but with differing degrees of complexity and risk. The same set of 27 tasks was presented in both phases; this is for the purpose of investigating the effect of experience on complexity preferences. However, the order in which the 27 tasks were presented changes between the two phases. Also, the way in which individual lotteries were presented, in terms of the order of the outcomes, changed between the two phases.

The random lottery incentive mechanism was employed. That is, at the end of the experiment one of the 54 tasks was randomly selected, and the lottery chosen by the subject in the selected task was played out to determine the earnings. Average earnings were around 11 pounds.

\section{Experimental Design}

Our experimental design is built on the pair of tasks used by Sonsino et al. (2002), presented in Table 1. We chose this task because it is the simplest that Sonsino et al. employ in their paper and, in particular, it is a single period task (some of their tasks involve multi-period lotteries, which we avoid).

\section{(Insert Tables $2 a$ and $2 b$ about here: if possible facing each other)}

Tables $2 a$ and $2 b$ show the 18 lotteries, other than the sure win SW of 107 points, that we used in the experiment. Six lotteries are simple (S), 6 are complex $(C)$, and 6 are very complex (VC). We derived the complex and the very complex lotteries from the simple lotteries using the same procedure as used in Sitzia and Zizzo (2011), which is an extension of the procedure used by Sonsino et al. (2002). An intuitive explanation of the procedure, in terms of averaging the outcomes from two independent plays of the same lottery, was provided in Section 2. The importance of this procedure is that it provides a way of making a lottery considerably more complex, while at the same time making it safer. This feature of the design - complexity and risk (sometimes) moving in opposite directions - is what enables us, in estimation, to separate the effect of complexity aversion from that risk aversion.

A formal explanation of the procedure is as follows. We will first show how we can generate, from a simple lottery $S_{a}$ with 3 outcomes, a complex lottery $C_{a}$ lottery with 9 outcomes, and then how we can generate a very complex lottery from the same simple lottery. Consider the following simple lottery:

$$
S_{a}=(\mathbf{p}, \mathbf{x})=\left(\left(p_{1} p_{2} p_{3}\right)^{\prime},\left(x_{1} x_{2} x_{3}\right)^{\prime}\right)
$$

\footnotetext{
${ }^{2}$ The instructions and questionnaire are provided in Appendix A.
} 
Note that $\mathbf{p}$ and $\mathbf{x}$ are $3 \times 1$ (column) vectors of probabilities and outcomes respectively. $\mathbf{p}$ is such that $\sum_{j=1}^{3} p_{j}=1$. A more complex lottery $C_{a}$ can be generated from $S_{a}$ using the following formula:

$$
C_{a}=\left(\operatorname{vec}\left(\mathbf{p} \mathbf{p}^{\prime}\right) ; \operatorname{vec}\left(\frac{1}{2} \mathbf{x i}_{3}{ }^{\prime}+\frac{1}{2} \mathbf{i}_{3} \mathbf{x}^{\prime}\right)\right)
$$

where $\mathbf{i}_{3}$ is a $3 \times 1$ (column) vector consisting only of ones, and $\operatorname{vec}(A)$ is the function that transforms a $n \times n$ matrix $A$ into $a n^{2} \times 1$ (column) vector consisting of the elements of $A$. Note that this complex lottery is equivalent to playing the simple lottery twice in succession and using the arithmetic mean outcome from the two plays as the outcome. Note also that the expected value of $C_{a}$ is the same as that of $S_{a}: E\left(C_{a}\right)=E\left(S_{a}\right)$.

From a complex lottery, using a procedure similar to the one just described, it is possible to create very complex lottery, with 27 outcomes. Let us consider the following complex lottery, $C_{b}$, similar to $C_{a}$ defined in (0), but with different (smaller) weights in the second argument:

$$
C_{b} \equiv(\mathbf{q}, \mathbf{y})=\left(\operatorname{vec}\left(\mathbf{p} \mathbf{p}^{\prime}\right) ; \operatorname{vec}\left(0.03 \mathbf{x i}_{3}{ }^{\prime}+0.07 \mathbf{i}_{3} \mathbf{x}^{\prime}\right)\right)
$$

Intuitively, $C_{b}$ is equivalent to two independent plays of $S_{a}$, with a weighted average being taken of the two outcomes, with weights 0.07 and 0.03 . However, note that, because the weights sum to $0.10, E\left(C_{b}\right)=0.1 \times E\left(S_{a}\right)$. We then combine $S_{a}$ and $C_{b}$, with the weights 0.9 and 1 , to obtain the very complex lottery $V C_{b}$ :

$$
v C_{b}=\left(\operatorname{vec}\left(\mathbf{q} \mathbf{p}^{\prime}\right) ; \operatorname{vec}\left(0.9 \mathbf{i}_{9} \mathbf{x}^{\prime}+\mathbf{y i}_{3}{ }^{\prime}\right)\right)
$$

where $\mathbf{i}_{9}$ is a $9 \times 1$ (column) vector consisting only of ones. Note that the two vectors defining $\mathrm{VC}_{\mathrm{b}}$ are of order $27 \times 1$, implying that this very complex lottery has 27 outcomes. Note also that $E\left(V C_{b}\right)=E\left(S_{a}\right)$.

Note that the procedure employed to generate complex and very complex lotteries does not guarantee that the actual numbers of outcomes are 9 and 27 respectively. The reason for this is that some outcomes may be the same. This is in fact the case for the complex lotteries (where the number of outcomes is 6 instead of 9 ) while for the very complex lotteries this does not happen and the number of outcomes is always 27.

Table 2 a shows the 3 simple lotteries, $\mathrm{S} 1, \mathrm{~S} 2$ and $\mathrm{S} 3$, and the lotteries derived from them. As previously noted, S3 is identical to one of the lotteries in Sonsino et al. (2002). S1 and S2 have been designed by us. They have the same expected value as S3 but more extreme lowest and highest outcomes, and also different middle outcomes. The procedures described above were used to obtain 3 complex (C1-C3) and 3 very complex (VC1-VC3) lotteries from the three simple lotteries (S1-S3).

Table $2 \mathrm{~b}$ shows our "safe" versions of the lotteries shown in Table $2 \mathrm{a}$. These are designated by the subscript "s" in the lottery name. The "s"-type lottery is derived by decreasing the spread of the extreme outcomes while leaving unchanged the middle outcome. Complex and very complex lotteries are then derived from the "s"-type simple lotteries using the procedures described above, and they are also given the "s" label. The "s"-type lotteries are only used in 
following choice tasks: $\mathrm{S}_{s}-\mathrm{S}, \mathrm{C}_{\mathrm{s}}-\mathrm{C}, \mathrm{VC}_{\mathrm{s}}-\mathrm{VC}$. The reason for using them in this way is to investigate whether risk attitude is influenced by task complexity. For example, if the propensity to choose $\mathrm{VC}_{s}$ over $\mathrm{VC}$ is higher than that of choosing $\mathrm{S}_{\mathrm{s}}$ over $\mathrm{S}$, this would simply indicate that subjects become more risk-averse when tasks become more complex.

Risk is sufficiently measured in our setting in terms of variance of the lotteries. For the time being it suffices to say that, for every simple lottery $S$, and the complex $(C)$ and very complex (VC) lotteries derived from it, it is the case that $0=V(S W)<V(C)<V(V C)<V(S)$.

\title{
4. Exploratory Data Analysis
}

In this section we will present some descriptive statistics, ahead of the more rigorous econometric modeling in the later sections.

Our sample consists of 80 subjects. Table 3 contains information about the sample. Almost $2 / 3$ of our subjects are female (last column in Table 3 ). We also notice (last row in Table 3 ) that about $37 \%$ are from China, $25 \%$ from the UK, $10 \%$ from Hong Kong and the remainder from other countries (the proportions are sufficiently small for these countries to be grouped together). It is interesting to see that most of our Chinese participants (83\%) are female, and this is true also for other nationalities except participants from Hong Kong, of which there is an equal number of males and females, and participants from the UK, of which $60 \%$ are male. The sample is spread over many different fields of study, with the most commonly observed fields being Accounting and Finance (11\% of sample) and Political, Social and International Studies (8\%). Finally, 53\% of our participants are enrolled in Bachelor's degrees, $38 \%$ in Master's degrees, with the remaining $9 \%$ in other postgraduate programs. The average age of our sample is 22.63 .

\section{(Insert Table 3 about here)}

There are 9 types of choice problem; these are defined in the second column of Table 4. All choice problems are represented as two lotteries separated by a hyphen, with the first lottery being the safer. Each of the 9 types consists of 3 particular choice problems (for example, type 1 is labelled SW-S, and consists of the 3 choice problems: SW-S1; SW-S2; SW-S3). This explains why the total number of tasks (within each phase) is 27.

The third column of Table 4 shows the total number of decisions made for each of the 9 types of choice problem. For most problem types ${ }^{3}$ this is 480 , being the product of: the number of different lotteries within the type (3); the number of subjects (80); and the number of phases (2).

The final column of Table 4 shows the proportion of decisions in which the safe choice is made. This information is also presented in Figure 1, with white bars used for tasks involving the sure win, black bars for tasks with lotteries of the same complexity level, and grey bars for the remaining tasks.

\author{
(Insert Table 4 about here) \\ (Insert Figure 1 about here)
}

\footnotetext{
${ }^{3}$ Note that task types 1 and 5 have only 440 observations, while all other task types have 480 . The reason for this difference is a minor programming bug that led to the loss of data on 40 of the 80 subjects' choices on each of the two choice problems: VC3-S3 and SW-S3.
} 
With respect to Table 4 and Figure 1, a number of comments are in order. Firstly, on more than $70 \%$ of occasions the sure win (SW) is preferred to the other lottery (white bars in Figure 1) and this does not appear to be affected by the complexity of the latter. When lotteries have the same complexity level (black bars), the safe lottery is chosen more frequently than the riskier one, indicating overall risk aversion, but these bars are not as high as the white bars, indicating that risk aversion is highest when one of the alternatives is a sure win. Note also that the black bars do not appear to be very different from each other, indicating that risk attitude does not change when task complexity increases. However, when lotteries of differing complexity levels are employed (grey bars), the proportion of safe choices seems to fall as the complexity level of the task (i.e. again taking both complexity levels into consideration) rises. Taken together, these results lead us to conclude that there is mixed evidence on the relationship between task complexity and risk aversion.

Some of the individual tasks can be paired in such a way as to allow pure tests of complexity aversion, in the manner of the example used in Section 2. The results of these tests are presented in Table 5.

\section{(Insert table 5 about here)}

The first two columns of Table 5 show the two tasks used in the test. For example, the first test compares the choices made in the two tasks SW-VC1 and VC1-S1. Using the same reasoning as used in the example of Section 2, if any subject switches from SW (the sure win) in the first task to $S 1$ (the simple lottery) in the second task, they are signalling complexity aversion, because $\mathrm{S} 1$ has a higher variance than VC1 despite being less complex. Such a subject is committing a "safe-to-risky" (SR) reversal. The direct test of complexity aversion simply compares, for each task-pair, the number of SR reversals with the number of RS reversals (shown in the $5^{\text {th }}$ and $6^{\text {th }}$ columns of Table 5). If the number of SR reversals is significantly greater, there is evidence of complexity aversion. As with the example in Section 2 , the McNemar test (Siegel and Castellan, 1988) is used to formalise this comparison. The last three columns of Table 5 show the test statistic, the p-value, and the conclusion of the test. We see that around half of the tests result in either evidence or strong evidence of complexity aversion. We also see that the evidence of complexity aversion appears to be weaker in the second phase (since p-values in the second round tend to be larger than the corresponding ones in the first round). The exception is the last two pairs, but here the evidence is stronger in the second round because the test is based on 80 observations instead of 40 . The difference between phases suggests a reduction in complexity aversion with experience.

These simple tests clearly provide some evidence of complexity aversion. In the next section we go a step further, by allowing complexity aversion to vary between individuals, and in particular we allow a positive proportion of the population to be complexity-neutral. This is achieved within the context of a finite-mixture random-effects model. The framework also allows estimation of the correlation between complexity aversion and risk aversion, and (formal) estimation of the effect of experience on complexity aversion. 


\section{Econometric Models of Complexity Aversion}

In this section we outline the econometric models whose estimates are reported in the next section. Technical details, such as the construction of the log-likelihood, are provided in Appendix B.

The modeling strategy is similar in some ways to that of Sonsino et al. (2002). Let the two lotteries in the choice problem be defined as:

riskier: $\quad(\mathbf{p}, \mathbf{x})=\left(p_{1} \cdots p_{J}, x_{1} \cdots x_{J}\right)$

safer: $\quad(\mathbf{q}, \mathbf{y})=\left(q_{1} \cdots q_{K}, y_{1} \cdots y_{K}\right)$

Importantly in the context of measuring complexity aversion, the two lotteries are permitted have different numbers of outcomes $(J$ and $K)$. The two lotteries have expected values:

$$
\begin{aligned}
& E(\mathbf{p}, \mathbf{x}) \equiv \mu_{(\mathbf{p}, \mathbf{x})}=\sum_{j=1}^{J} p_{j} x_{j} \\
& E(\mathbf{q}, \mathbf{y}) \equiv \mu_{(\mathbf{q}, \mathbf{y})}=\sum_{j=1}^{K} q_{j} y_{j}
\end{aligned}
$$

and variances:

$$
\begin{aligned}
& V(\mathbf{p}, \mathbf{x}) \equiv \sigma_{(\mathbf{p}, \mathbf{x})}{ }^{2}=\sum_{j=1}^{J} p_{j}\left(x_{j}-\mu_{(\mathbf{p}, \mathbf{x})}\right)^{2} \\
& V(\mathbf{q}, \mathbf{y}) \equiv \sigma_{(\mathbf{q}, \mathbf{y})}{ }^{2}=\sum_{j=1}^{K} q_{j}\left(y_{j}-\mu_{(\mathbf{q}, \mathbf{y})}\right)^{2}
\end{aligned}
$$

The riskier lottery is defined as the one with the higher variance. Hence:

$$
\sigma_{(\mathbf{p}, \mathbf{x})}{ }^{2}>\sigma_{(\mathbf{q}, \mathbf{y})}{ }^{2}
$$

Since all lotteries have the same mean, but differing variances, a natural and convenient basic framework in which to operate is that of the mean-variance utility function. We will assume that the choice between two lotteries is based on the difference between the (mean-variance) utilities of the two lotteries. Within this framework, we assume the existence of four types. Indexing individuals by $\mathrm{i}$, and letting $U_{i}$ be the utility function for individual $i$, the four types are defined as follows:

Type 1 (RN and CN):

$$
U_{i, 1}(\mathbf{p}, \mathbf{x})=\mu_{(\mathbf{p}, \mathbf{x})}
$$

Type $2(\mathrm{CN})$ :

$$
U_{i, 2}(\mathbf{p}, \mathbf{x})=\mu_{(\mathbf{p}, \mathbf{x})}-\alpha_{i} \sigma_{(\mathbf{p}, \mathbf{x})}{ }^{2}
$$

Type $3(\mathrm{RN})$ :

$$
U_{i, 3}(\mathbf{p}, \mathbf{x})=\mu_{(\mathbf{p}, \mathbf{x})}-\gamma_{i} C(\mathbf{p}, \mathbf{x})
$$

Type 4 (neither RN nor CN): $U_{i, 4}(\mathbf{p}, \mathbf{x})=\mu_{(\mathbf{p}, \mathbf{x})}-\alpha_{i} \sigma_{(\mathbf{p}, \mathbf{x})}{ }^{2}-\gamma_{i} C(\mathbf{p}, \mathbf{x})$ 
RN stands for "risk-neutral", that is, non-responsive to variance; CN stands for "complexityneutral", that is, non-responsive to complexity. The parameter $\alpha$ is closely related to the coefficient of absolute risk aversion (see Chavas and Pope, 1983). $C(\mathbf{p}, \mathbf{x})$ is the chosen measure of the complexity of the lottery (operationalized as: $\mathrm{C}=0$ for Sure Win; $\mathrm{C}=1$ for Simple; $\mathrm{C}=2$ for complex; $\mathrm{C}=3$ for very complex). $\gamma$ is a parameter representing the degree of complexity aversion. We expect $\gamma>0$. Note further that we will be assuming between-subject heterogeneity both in risk aversion $(\alpha)$ and in complexity aversion $(\gamma)$; hence the $i$-subscripts on these parameters.

The distribution we will assume for risk aversion $(\alpha)$ and in complexity aversion $(\gamma)$ is:

$$
\left(\begin{array}{l}
\alpha \\
\gamma
\end{array}\right) \sim N\left[\left(\begin{array}{l}
\theta_{1} \\
\theta_{2}
\end{array}\right),\left(\begin{array}{cc}
\eta_{1}^{2} & \rho \eta_{1} \eta_{2} \\
\rho \eta_{1} \eta_{2} & \eta_{2}^{2}
\end{array}\right)\right]
$$

Hence the parameters of principal interest are the two means, $\theta_{1}$ and $\theta_{2}$, the two standard deviations, $\eta_{1}$ and $\eta_{2}$, and the correlation $\rho$. We shall also allow the means $\left(\theta_{1}\right.$ and $\left.\theta_{2}\right)$ to depend on experience and subject characteristics. Since there are four types (defined in 4a$4 d$ ), we also require the estimation of a set of mixing proportions which we shall label $\pi_{1}-\pi_{4}$. There is also a parameter $\varphi$, whose sign will indicate whether the magnitude of computational errors increases $(\varphi>0)$ or decreases $(\varphi<0)$ over the course of the experiment. The former will be interpreted as a "boredom" effect, and the latter as an "experience" effect.

Finally, we will allow for the possibility that agents do not take the stated probabilities at face value. ${ }^{4}$ One hypothesis that is of particular relevance for compex lotteries is that agents act as Bayesians, and view the prior probability of each outcome as $1 / J$ (where $J$ is the number of outcomes in the lottery). A way of modelling this is to assume that agents act on transformed probabilties $\left(\tilde{p}_{j}\right)$, given by:

$$
\tilde{p}_{j}=\frac{\delta \frac{1}{J}+p_{j}}{\delta+1} \quad j=1, \cdots, J ; J>1
$$

(6) is, in fact, a form of Prospective Reference Theory (Viscusi, 1989). The important quantity in (6) is the parameter $\delta$. If $\delta=0$, probabilities are correctly interpreted ( $\tilde{p}_{j}=p_{j}$ for all $j$ ). If $\delta$ is large and positive, all probabilities ${ }^{5}$ become equal $\left(\tilde{p}_{j} \rightarrow(1 / J)\right.$ for all $\left.J\right)$. Hence, in order to test this form of Prospective Reference Theory, we shall incorporate (6) in our model, and test the null hypothesis $\delta=0$ against the alternative $\delta>0$.

The likelihood function is constructed by appending a stochastic term to the utility difference pertaining to each choice problem and deducing choice probabilities. Estimation is performed using the method of Maximum Simulated Likelihood (MSL) (Train, 2003). See Appendix B for

\footnotetext{
${ }^{4}$ We thank Kip Viscusi for suggesting this extension to our model.

${ }^{5}$ It is important to realise that (6) does not apply when $J=1$. In this case, $\tilde{p}_{1}=p_{1}=1$; that is, the probability of the certain outcome is correctly interpreted as 1 .
} 
further details. Maximization of the simulated likelihood function is performed using the $\mathrm{ml}$ routine in STATA. ${ }^{6}$

\section{Estimation Results}

In the data, all money amounts are divided by 107 , so that the expected value of all lotteries is 1 .

Six sets of results are presented in Tables $6 \mathrm{a}$ and $6 \mathrm{~b}$. All sets of results are from maximisation of a log-likelihood function based on (A6) in Appendix B. We start off with the "homogeneous" model (Model 1), in which it is assumed that all individuals have the same risk aversion and complexity aversion. These are estimated respectively as +2.964 and +0.031 , both significantly greater than zero, implying (overall) significant risk aversion (RA) and complexity aversion (CA). We then estimate the finite mixture model (Model 2) which leads to a major improvement statistically, but for which the results are dubious. Both RA and CA are estimated as very large, but from the mixing proportions, we see that a large proportion of subjects are irresponsive to risk and/or complexity. For example, the proportion who are complexityneutral is $0.289+0.554=0.843$. The problem with this model is that all subjects who respond to complexity are being "forced" to have the same CA, with the result that a very small proportion are identified as extremely complexity-averse, with the remainder being complexityneutral. This leads us to Model 3 , which is the finite mixture random effects model. Here, we notice firstly that the two heterogeneity parameters $\left(\eta_{1}\right.$ and $\left.\eta_{2}\right)$ are both strongly positive, indicating that RA and CA both vary continuously between subjects who are responsive. Secondly, we note that the parameter $\rho$ is significantly negative, indicating a negative association between RA and CA: subjects who are more risk-averse tend to be less complexity-averse. Thirdly, we notice that the proportion $\left(\pi_{1}\right)$ of subjects who are of type 1 has fallen all the way to zero. This is a promising result, since it implies that there are no subjects in the sample who respond to neither risk nor complexity. The change in the estimate of this parameter between Models 2 and 3 is a direct result of the introduction of continuous heterogeneity in RA and CA. Models 4-6 exclude types 1 and 3 because of their apparent absence from the population.

\section{(Insert Tables 6a and 6b about here, if possible facing each other)}

Model 4 introduces the effect of experience (represented by position of task in sequence, $\tau$ ) in two places: CA and the error variance are both assumed to depend on experience. The effect of experience on RA was found to be insignificant. ${ }^{7}$ Experience does have a significantly negative effect on CA, with the estimated equation (from Model 4) being:

$$
\hat{\theta}_{2}(\tau)=0.224-0.006 \tau
$$

(7) essentially implies that a typical (type 4, i.e. not complexity-neutral) subject is moderately averse to complexity at the start of the experiment, but becomes less complexity-averse in the course of the experiment, and becomes complexity-loving after 37 tasks (recall that there are a total of 54 tasks in the experiment). Model 4 also allows the computational error variance, which represents the behavioral noise in our models, to change with experience, in

\footnotetext{
${ }^{6}$ The STATA code used for estimation is available from the authors on request.

7 When $\tau$ is added as a determinant of risk aversion to Model 4 , its asymptotic t-statistic is -0.40 and the $p$-value is 0.69 .
} 
accordance with (A3) in Appendix B. The significantly negative estimate of the parameter $\varphi$ indicates that this error variance falls with experience, and this is consistent with an experience effect rather than a boredom effect. Another feature of model is that the correlation $(\rho)$ between RA and CA is again negative, but not significantly different from zero. It appears that the significant value seen in Model 3 was the manifestation of a misspecification bias caused by neglecting the effect of experience.

Models 5 and 6 introduce some subject characteristics to the CA equation. These variables were found to be insignificant when also added to the RA equation. ${ }^{8}$ We will return to the subject characteristics shortly. Model 6 introduces the reference-dependence parameter $\delta$ defined in (6). We see that the estimate of $\delta$ is positive and significant. We also see that the AICs clearly indicate that Model 6 is the best of the six models. However, the estimate of $\delta$ is small in magnitude at +0.020 . The interpretation, in accordance with (6), is that subjects perceive probabilities as slightly different from the true probabilities, in a direction towards an equal probability for all outcomes in the lottery.

Returning to the subject characteristics, and focusing on the results of Model 6, males appear to be less complexity-averse than females, although this effect is not statistically significant. Age and "non-UK" both have positive and (in the first case) significant effects on complexity aversion. In the sample, $66 \%$ are female, $75 \%$ are "non-UK", and the mean of age is around 23 (see Table 3). We will therefore use Model 6 to obtain an equation similar to (7) for a 23year-old, female, non-UK student. The equation is:

$$
\hat{\theta}_{2}(\tau)=+0.423-0.007 \tau
$$

(8) implies that, for this type of subject, CA reaches zero after 60 tasks (i.e. roughly by the end of the experiment).

Based on Model 6, it seems that there exist subjects (around $33 \%$ of the population) who are complexity-neutral throughout, while the remaining $67 \%$ are (on average) complexity-averse, with complexity aversion falling fairly rapidly with experience, to the extent that they are (on average) almost complexity-neutral by the end of the experiment.

Although we already have an estimate of the proportion of the population who are complexityneutral (33\%), we can go further by classifying individual subjects on the basis of posterior estimation. Figure 2 shows a frequency histogram of the posterior probability of being type 2 (complexity-neutral), obtained using (A9) in Appendix B, following estimation of Model 6 . We see that, although a large number of subjects appear very unlikely to be complexity-neutral, few have a very high posterior probability of being so. In order to be true to our estimate of $33 \%$ being complexity-neutral, it seems sensible to classify as complexity-neutral the $33 \%$ of subjects (26 out of 80 ) with the highest posterior probabilities of being so. The cut-off appears to be $0.51 ; 26$ subjects (33\%) have posterior probabilities of greater than 0.51 , and we shall classify these 26 as complexity-neutral.

\section{(Insert Figure 2 about here.)}

\footnotetext{
${ }^{8}$ When the three explanatory variables male, (age-18), and non_UK are all added to the risk-aversion equation in Model 5, the LogL rises very slightly to -2410.25 , but the associated AIC is 4848.5 , which is vastly inferior to Model 5 .
} 
Figure 3 shows posterior estimates of $\alpha$ and $\gamma$ obtained from Model 6 , using a formula such as (A10) in Appendix B. Observations on the horizontal axis are the 26 subjects whose posterior type probabilities indicate that they are of type $2(\mathrm{CN})$, and hence with $\gamma=0$. Based on the information displayed in Figure 3, Table 7 classifies subjects by risk and complexity preferences. In particular, we find that $17 \%, 33 \%$ and $50 \%$ of the subjects are complexityloving, neutral and averse, respectively.

\section{(Insert Figure 3 and Table 7 about here.)}

\section{Conclusion}

An understanding of complexity preferences is important both in assessing the relevance of theoretical models that assume complexity-averse populations, and in evaluating previous experimental evidence on the phenomenon.

Using lotteries that vary in terms of both complexity and variance, we have conducted a choice experiment, and then estimated a model which allows heterogeneity in both risk aversion and complexity aversion.

Both types of heterogeneity are seen to be important. The need for a finite mixture approach, with different types of subject, is clear, since the population seems to divide between subjects who respond to complexity, and those who are complexity-neutral. However, we have found that a model which only assumes a mixture of types, while far superior to the homogeneous model, gives misleading results, because of the failure to allow variation in complexity preferences. Our preferred model was one which contained both a finite mixture structure, and also continuous between-subject variation in both complexity aversion and risk aversion. For this model - the finite-mixture random-effects model - the results seemed much more plausible. Further useful extensions of the model included allowance for the effects of experience and subject characteristics, and also the introduction of a parameter capturing prospective reference theory, which allows for misperceptions of probabilities in the direction of equal probabilities for all outcomes.

The main findings are that around $33 \%$ of the population are complexity neutral. Of the $67 \%$ who respond to complexity, the typical subject displays a moderate level of complexity aversion, but in the course of the experiment, this complexity aversion falls all the way towards zero, implying complexity neutrality at the end of the experiment. These results are consistent with the results of simple non-parametric tests for complexity aversion reported in Section 4. There is also an effect of experience in reducing the variance of computational error. Both of the experience effects may be interpreted as forms of learning. These results have important implications in real life decisions. When decisions are infrequent ${ }^{9}$ (such as buying a house, choosing a retirement plan or a health plan, choosing a tariff plan) and learning opportunities are scarce, decision makers are more likely to be complexity-averse. They might avoid making a decision altogether; they might stick with the default option (lyengar and Lepper 2000, Thaler and Sunstein 2007); they might procrastinate; or they might choose a simple but worse option. In any of these cases, there are opportunites for policies to be designed to make the decision environment simpler and hence to 'nudge' individuals to better outcomes. However, when decisions are complex but made frequently, decision makers may become

\footnotetext{
${ }^{9}$ Changing an energy tariff, a mobile phone tariff, or a bank account are decisions that can in principle be made frequently. However, in practice such decisions are typically seen to be quite infrequent (Sitzia et al. 2014). In addition, frequent changes in tariffs are likely to impede learning. Hence we expect complexity aversion to play an important role in these situations.
} 
accustomed to the decision environment, hence becoming less complexity-averse, and reducing the need for policy interventions. We further conjecture that, if feedback on decisions were available (unlike in our experiment), one could expect a faster convergence towards complexity neutrality, better decisions, and therefore less need for policy intervention (Thaler and Sunstein, 2003). We leave this for future research.

Complexity aversion also depends on subject characteristics such as age and nationality. It is very interesting that risk aversion, in contrast, does not appear to depend on either experience or subject characteristics.

We found some evidence of a negative association between risk aversion and complexity aversion, although the correlation parameter representing this association was estimated as positive but insignificant in our preferred model.

When we use posterior estimation to classify subjects, we find that around $17 \%$ of subjects are complexity-loving. This is an important finding, of which theorists who construct models on the assumption of complexity aversion should be aware. This is particularly important if the theoretical models are intended to represent the behavior of agents with repetition and learning opportunities.

In the presence of inexperienced agents, our results suggest that making a general assumption of complexity aversion may indeed be an adequate way of reflecting aggregate behavior, since the proportion of complexity-averse agents is likely to be larger than that of complexity-loving agents. That said, further research should be targeted at gaining a better understanding of complexity-loving preferences. 


\section{REFERENCES}

Bruce, C., \& Johnson, J. E. V. (1996). Decision-making under risk: The effect of complexity on performance. Psychological Reports, 79, 67-76.

Chavas, J. P., \& Pope, R., (1982). Hedging and production decisions under a linear meanvariance preference function. Western Journal of Agricultural Economics, 7(1), 99110.

Ellison, G., \& Ellison, S. F. (2004). Search, obfuscation, and price elasticities on the internet. Discussion Paper, MIT and NBER.

Fischbacher, U. (2007). z-Tree: Zurich toolbox for ready-made economic experiments. Experimental Economics, 10, 171-178.

Gale, D., \& Sabourian, H. (2005). Complexity and competition. Econometrica, 73(3), 739-769.

Garrod, L., Hviid, M., Loomes, G., \& Waddams Price, C. (2009). Competition remedies in consumer markets. Loyola Consumer Law Review, 21(4), 439-495.

Huck, S., \& Weizsäcker, G. (1999). Risk, complexity, and deviations from expected-value maximization: Results of a lottery choice experiment. Journal of Economic Psychology, 20(6), 699-715.

Humphrey, S. J. (1995). Regret aversion or event-splitting effects? More evidence under risk and uncertainty. Journal of Risk and Uncertainty, 11(3), 263-274.

Humphrey, S. J. (2001). Are event-splitting effects actually boundary effects? Journal of Risk and Uncertainty, 22(1), 79-93.

lyengar, S. S., \& Lepper, M. (2000). When choice is demotivating: Can one desire too much of a good thing? Journal of Personality and Social Psychology, 79, 995-1006.

Mador, G., Sonsino, D., \& Benzion, U. (2000). On complexity and lotteries evaluation-Three experimental observations. Journal of Economic Psychology, 21(6), 625-637.

Rouse, A. (2008). Consumers confused by modern cars. http://www.streetdirectory.com/travel guide/162716/shopping/consumers confused by modern cars.html.

Siegel, S., \& Castellan, N. J. (1988). Non-parametric statistics for the behavioral sciences. $2^{\text {nd }}$ edition, McGraw Hill.

Sitzia, S., \& Zizzo, D. J. (2011). Does product complexity matter for competition in experimental retail markets? Theory and Decision, 70(1), 65-82.

Sitzia, S., Zheng, J., \& Zizzo, D. J. (2012). Complexity and smart nudges with inattentive consumers. Social Science Research Network Discussion Paper.

Sonsino, D., Benzion, U., \& Mador, G. (2002). The complexity effects on choice with uncertainty-Experimental evidence. The Economic Journal, 112(482), 936-965. 
Starmer, C., \& Sugden, R. (1993). Testing for juxtaposition and event-splitting effects. Journal of Risk and Uncertainty, 6(3), 235-254.

Stodder, J. (1997). Complexity aversion: Simplification in the Herrnstein and Allais behaviors. Eastern Economic Journal, 23(1), 1-31.

Sunstein, C. R., \& Thaler, R. H. (2003). Libertarian paternalism is not an oxymoron. University of Chicago Law Review, 70, 1159-202.

Thaler, R. H., \& Sunstein, C. R. (2008). Nudge: improving decisions about health, wealth, and happiness. New Haven, CT: Yale University Press.

Train, K. E. (2003). Discrete choice methods with simulation. Cambridge University Press.

Viscusi, W. K. (1989). Prospective reference theory: Toward an explanation of the paradoxes. Journal of Risk and Uncertainty, 2(3), 235-263.

Weber, B. J. (2007). The effects of losses and event splitting on the Allais paradox. Judgment in Decision Making, 2(2), 115-125.

Wilcox, N. (1993). Lottery choice: Incentives, complexity and decision time. Economic Journal, 103(421), 1397-1417.

Zuckerman, M. (2006). Sensation seeking and risky behavior. American Psychological Association, http://dx.doi.org/10.1037/11555-002.

Zuckerman, M. (1979). Sensation seeking: Beyond the optimal level of arousal. Hillsdale, NJ: L. Erlbaum Associates, Inc. 


\section{APPENDIX A}

\section{Experimental Instructions}

In the course of this experiment, over a number of periods you will be asked to choose between lotteries that pay returns in experimental points with given probabilities.

In the table below you see an example of a lottery of the kind a unit of which you can choose each period:

\begin{tabular}{|c|c|c|}
\hline OUTCOME & PROBABILITY & RETURN \\
\hline 1 & $35 \%$ & 50 \\
2 & $15 \%$ & 15 \\
3 & $18 \%$ & 80 \\
4 & $22 \%$ & 139 \\
5 & $10 \%$ & 10 \\
\hline
\end{tabular}

Table 1: Example of Product

In this example, the lottery will give you the chance to earn the following returns at the end of the experiment: 50 points with a probability of $35 \%$ (Outcome 1 ); 15 points with a probability of $15 \%$ (Outcome 2); 80 points with a probability of $18 \%$ (Outcome 3 ); and so on.

If you see a lottery which provides a given return with a probability of $100 \%$, this means that, if you choose this lottery, you will get that return for sure.

\section{Earnings}

At the end of the experiment the computer will randomly select one of the periods that will be used to determine your earnings. The computer will then randomly select one outcome of the lottery you have chosen in that period based on the probabilities. This outcome determines the return of that lottery and the points you have earned in the experiment. Every 10 points you own are converted into 1 pound, and so for example 80 points are worth 8 pounds.

Before starting to take decisions, we ask you to fill the enclosed questionnaire, with the only purpose of checking whether you have understood these instructions. Raise your hand when you have completed the questionnaire.

\section{Questionnaire}

1) Your earnings in the experiment are the sum of your earnings each period?

Yes

No

2) Consider the example lottery in Table 1 . What is the probability of obtaining a return of 80 ?

\section{PLEASE RAISE YOUR HAND WHEN YOU HAVE FINISHED. THANK YOU FOR ANSWERING THE QUESTIONNAIRE}




\section{APPENDIX B}

Construction of log-likelihood function.

For a given subject (i), who is of type j, facing a given choice problem (t), we define:

$$
\nabla U_{i t, j}\left(\alpha_{i}, \gamma_{i}\right)=U_{i, j}\left(\mathbf{p}_{\mathbf{t}}, \mathbf{x}_{\mathbf{t}}\right)-U_{i, j}\left(\mathbf{q}_{\mathbf{t}}, \mathbf{y}_{\mathbf{t}}\right)
$$

where the two terms on the right are defined according to one of $(4 a)-(4 d)$, depending on the type of subject i.

Subject $i$ (of type j) chooses lottery $\left(\mathbf{q}_{t}, \mathbf{y}_{\mathbf{t}}\right)$ over lottery $\left(\mathbf{p}_{\mathbf{t}}, \mathbf{x}_{\mathbf{t}}\right)$ if the following inequality holds:

$$
\nabla \bigcup_{i t, j}\left(\alpha_{i}, \gamma_{i}\right)+\varepsilon_{i t}<0
$$

where $\varepsilon_{i t}$ is a stochastic term with distribution:

$$
\varepsilon_{i t} \sim N\left(0, \exp \left(\varphi \tau_{i t}\right)\right)
$$

The stochastic term $\varepsilon$ may be interpreted as computational error, and its variance is assumed to depend on $\tau_{\text {it, }}$ which is the position of task $t$ in subject is sequence of tasks $(\tau=1, \ldots, 54)$. According to (A3), this variance is 1 at the start of the experiment-a normalization that is required for identification of the model. A negative sign of the parameter $\varphi$ will indicate that the magnitude of errors tends to decrease as the experiment progresses (an experience effect), while a positive sign will indicate an increase in errors over the course of the experiment (a boredom effect).

We define the binary variable $y_{i t}$ as follows. $y_{i t}=+1$ if subject i chooses $\left(\mathbf{q}_{t}, \mathbf{y}_{\mathbf{t}}\right)$ over $\left(\mathbf{p}_{\mathbf{t}}, \mathbf{x}_{\mathbf{t}}\right)$; $y_{\text {it }}=-1$ if subject i chooses $\left(\mathbf{p}_{\mathbf{t}}, \mathbf{x}_{\mathbf{t}}\right)$ over $\left(\mathbf{q}_{\mathbf{t}}, \mathbf{y}_{\mathbf{t}}\right)$. By (A2) and (A3):

$$
P\left(y_{i t}=1 \mid i \in j\right)=P\left[\nabla U_{i t, j}\left(\alpha_{i}, \gamma_{i}\right)+\varepsilon_{i t}<0\right]=\Phi\left(-\frac{\nabla U_{i t, j}\left(\alpha_{i}, \gamma_{i}\right)}{\sqrt{\exp \left(\varphi \tau_{i t}\right)}}\right) \quad i=1, \cdots, n \quad t=1, \cdots, T
$$

Where " $i \in j$ " denotes that subject $i$ is of type $j$. Note that we are assuming that there are in total $\mathrm{n}$ subjects each facing $\mathrm{T}$ choice problems.

As noted in (5) in the main text, we are assuming between-subject heterogeneity both in risk aversion $(\alpha)$ and in complexity aversion $(\gamma)$, and their joint distribution will be assumed to be:

$$
\left(\begin{array}{l}
\alpha \\
\gamma
\end{array}\right) \sim N\left[\left(\begin{array}{c}
\theta_{1} \\
\theta_{2}
\end{array}\right),\left(\begin{array}{cc}
\eta_{1}^{2} & \rho \eta_{1} \eta_{2} \\
\rho \eta_{1} \eta_{2} & \eta_{2}^{2}
\end{array}\right)\right]
$$

$\rho$ is the correlation coefficient between risk aversion and complexity aversion. 
The likelihood contribution associated with subject $\mathrm{i}$ is:

$L_{i}=\iint_{\alpha} \sum_{j=1}^{4} \pi_{j} \prod_{t=1}^{T} \Phi\left(-y_{i t} \times \frac{\nabla U_{i t, j}\left(\alpha_{i}, \gamma_{i}\right)}{\sqrt{\exp \left(\varphi \tau_{i t}\right)}}\right) f\left(\alpha, \gamma ; \theta_{1}, \eta_{1}, \theta_{2}, \eta_{2}, \rho\right) d \gamma d \alpha$

where $f\left(\alpha, \gamma ; \theta_{1}, \eta_{1}, \theta_{2}, \eta_{2}, \rho\right)$ is the joint density function of $\alpha$ and $\gamma$, and the parameters $\pi_{\mathrm{j}}$, $\mathrm{j}=1 . .4$ are mixing proportions, representing the proportion of the population who are of each type.

A further extension to the model allows risk aversion and complexity aversion to depend on experience within the experiment, and also on demographics. In Models 5 and 6, we assume that:

$$
\begin{aligned}
& \theta_{1, i t}=\theta_{10}+\theta_{11} \tau_{i t} \\
& \theta_{2, i t}=\theta_{20}+\theta_{21} \tau_{i t}+\theta_{22} \text { male }_{i}+\theta_{23}(\text { age }-18)_{i}+\theta_{24} \text { non_u }_{i}
\end{aligned}
$$

where $\tau_{\text {it }}$ is, as in (A3), the position of task $t$ in subject is sequence of tasks $(\tau=1, \ldots, 54)$. (A7) allows the means (over the population) of the coefficients of risk aversion and complexity aversion, to change with experience and demographics. For example, the mean of the coefficient of complexity aversion (for an 18-year-old, female, UK subject) is $\theta_{20}$ at the start of the sequence, and changes by an amount $\theta_{21}$ with each task undertaken.

A yet further extension allows misinterpretation of stated probabilities in accordance with Prospective Reference Theory. If the transformed probabilities defined in (6) are used in place of true probabilities, we may rewrite (A1) as:

$$
\tilde{\nabla} U_{i t, j}\left(\alpha_{i}, \gamma_{i}\right)=U_{i, j}\left(\tilde{\mathbf{p}}_{\mathbf{t}}, \mathbf{x}_{\mathbf{t}}\right)-U_{i, j}\left(\tilde{\mathbf{q}}_{\mathbf{t}}, \mathbf{y}_{\mathbf{t}}\right)
$$

where $\tilde{\mathbf{p}}_{\mathbf{t}}$ and $\tilde{\mathbf{q}}_{\mathbf{t}}$ are vectors of transformed probabilities. Then the likelihood function is constructed using $\tilde{\nabla} U_{i t, j}$ defined in (A8) in place of $\nabla U_{i t, j}$ in (A6).

If Prospective Reference Theory were assumed, and specification (A7) were used, there would be a total of 15 parameters to estimate: $\theta_{10}, \theta_{11}, \eta_{1}, \theta_{20}, \theta_{21}, \theta_{22}, \theta_{23}, \theta_{24}, \eta_{2}, \rho, \varphi, \delta$, and three of the four mixing proportions: $\pi_{1}, \pi_{2}, \pi_{3}$. The models we in fact estimate contain somewhat fewer parameters since they are restricted versions of the general model.

Estimation is performed using the method of Maximum Simulated Likelihood (MSL) (Train 2003). This requires the use of two sets of Halton draws, which, when converted to normality, represent simulated realizations of the random parameters $\alpha$ and $\gamma$. Maximization of the simulated likelihood function is performed using the $\mathrm{ml}$ routine in STATA. ${ }^{10}$

10 The STATA code used for estimation is available from the authors on request. 
Having estimated the model, posterior type probabilities can be obtained, and also posterior subject-specific estimates of risk aversion and complexity aversion. To obtain the posterior type probabilities, we use a version of Bayes' rule:

$$
P\left(i \in j \mid y_{i 1} \cdots y_{i T}\right)=\frac{\hat{\pi}_{j} \iint_{\alpha} \prod_{t=1}^{T} \Phi\left(-y_{i t} \times \frac{\nabla U_{i t, j}(\alpha, \gamma)}{\sqrt{\exp \left(\varphi \tau_{i t}\right)}}\right) f\left(\alpha, \gamma ; \hat{\theta}_{1}, \hat{\eta}_{1}, \hat{\theta}_{2}, \hat{\eta}_{2}, \hat{\rho}\right) d \gamma d \alpha}{\hat{L}_{i}}
$$

Where hats indicate MLEs, and $\hat{L}_{i}$ is the likelihood contribution for subject $i$ as defined in (A6), with parameters replaced by MLEs.

To obtain the posterior subject-specific estimates of risk aversion, we use:

$$
\hat{\alpha}_{i}=E\left(\alpha_{i} \mid y_{i 1} \cdots y_{i T}\right)=\frac{\iint_{\gamma} \alpha \prod_{t=1}^{T} \Phi\left(-y_{i t} \times \frac{\nabla U_{i t, 4}(\alpha, \gamma)}{\sqrt{\exp \left(\varphi \tau_{i t}\right)}}\right) f\left(\alpha, \gamma ; \hat{\theta}_{1}, \hat{\eta}_{1}, \hat{\theta}_{2}, \hat{\eta}_{2}\right) d \gamma d \alpha}{\iint_{\alpha} \prod_{t=1}^{T} \Phi\left(-y_{i t} \times \frac{\nabla U_{i t, 4}(\alpha, \gamma)}{\sqrt{\exp \left(\varphi \tau_{i t}\right)}}\right) f\left(\alpha, \gamma ; \hat{\theta}_{1}, \hat{\eta}_{1}, \hat{\theta}_{2}, \hat{\eta}_{2}\right) d \gamma d \alpha}
$$

Note that we are conditioning on the subject being of type 4 (responsive to both variance and complexity) when computing their posterior risk aversion. Posterior subject-specific estimates of complexity aversion, denoted $\hat{\gamma}_{i}$, are computed using a formula similar to (A10). 
Table 1 - Sonsino et al. Binary Choices

\begin{tabular}{ccc}
\hline \hline & SW & \\
Outcome & Probability & Return \\
\hline 1 & 1 & 107 \\
\hline \hline
\end{tabular}

\begin{tabular}{ccc}
\hline \hline & C3 & \\
Return & Probability & Return \\
\hline 1 & 0.16 & 80 \\
2 & 0.24 & 90 \\
3 & 0.09 & 100 \\
4 & 0.24 & 115 \\
5 & 0.18 & 125 \\
6 & 0.09 & 150 \\
\hline \hline
\end{tabular}

\section{Choice}

1

\begin{tabular}{ccc}
\hline \hline & S3 & \\
Outcome & Probability & Return \\
\hline 1 & 0.4 & 80 \\
2 & 0.3 & 100 \\
3 & 0.3 & 150 \\
\hline \hline
\end{tabular}

Choice

2

\begin{tabular}{ccc}
\hline \hline & S3 & \\
Outcome & Probability & Return \\
\hline 1 & 0.4 & 80 \\
2 & 0.3 & 100 \\
3 & 0.3 & 150 \\
\hline \hline
\end{tabular}


Table 2a. Lotteries Employed in the Experiment

\begin{tabular}{|c|c|c|c|c|c|c|}
\hline & S1 & & S2 & & S3 & \\
\hline Outcome & Probability & Return & Probability & Return & Probability & Return \\
\hline 1 & 0.5 & 57 & 0.5 & 50 & 0.4 & 80 \\
\hline 2 & 0.2 & 112 & 0.2 & 113 & 0.3 & 100 \\
\hline \multirow[t]{2}{*}{3} & 0.3 & 187 & 0.3 & 198 & 0.3 & 150 \\
\hline & $\mathrm{C} 1$ & & $\mathrm{C} 2$ & & C3 & \\
\hline Outcome & Probability & Return & Probability & Return & Probability & Return \\
\hline 1 & 0.25 & 57 & 0.25 & 50 & 0.16 & 80 \\
\hline 2 & 0.2 & 84.5 & 0.2 & 81.5 & 0.24 & 90 \\
\hline 3 & 0.04 & 112 & 0.04 & 113 & 0.09 & 100 \\
\hline 4 & 0.3 & 122 & 0.3 & 124 & 0.24 & 115 \\
\hline 5 & 0.12 & 149.5 & 0.12 & 155.5 & 0.18 & 125 \\
\hline \multirow[t]{2}{*}{6} & 0.09 & 187 & 0.09 & 198 & 0.09 & 150 \\
\hline & VC1 & & VC2 & & VC3 & \\
\hline Outcome & Probability & Return & Probability & Return & Probability & Return \\
\hline 1 & 0.125 & 57 & 0.125 & 50 & 0.064 & 80 \\
\hline 2 & 0.05 & 58.65 & 0.05 & 51.89 & 0.048 & 80.6 \\
\hline 3 & 0.05 & 60.85 & 0.05 & 54.41 & 0.048 & 81.4 \\
\hline 4 & 0.075 & 60.9 & 0.075 & 54.44 & 0.036 & 82 \\
\hline 5 & 0.02 & 62.5 & 0.02 & 56.3 & 0.048 & 82.1 \\
\hline 6 & 0.03 & 64.75 & 0.03 & 58.85 & 0.036 & 83.5 \\
\hline 7 & 0.075 & 66.1 & 0.075 & 60.36 & 0.048 & 84.9 \\
\hline 8 & 0.03 & 67.75 & 0.03 & 62.25 & 0.036 & 85.5 \\
\hline 9 & 0.045 & 70 & 0.045 & 64.8 & 0.036 & 87 \\
\hline 10 & 0.05 & 106.5 & 0.05 & 106.7 & 0.048 & 98 \\
\hline 11 & 0.02 & 108.15 & 0.02 & 108.59 & 0.036 & 98.6 \\
\hline 12 & 0.02 & 110.35 & 0.02 & 111.11 & 0.036 & 99.4 \\
\hline 13 & 0.03 & 110.4 & 0.03 & 111.14 & 0.027 & 100 \\
\hline 14 & 0.008 & 112 & 0.008 & 113 & 0.036 & 100.1 \\
\hline 15 & 0.012 & 114.25 & 0.012 & 115.55 & 0.027 & 101.5 \\
\hline 16 & 0.03 & 115.6 & 0.03 & 117.06 & 0.036 & 102.9 \\
\hline 17 & 0.012 & 117.25 & 0.012 & 118.95 & 0.027 & 103.5 \\
\hline 18 & 0.018 & 119.5 & 0.018 & 121.5 & 0.027 & 105 \\
\hline 19 & 0.075 & 174 & 0.075 & 183.2 & 0.048 & 143 \\
\hline 20 & 0.03 & 175.65 & 0.03 & 185.09 & 0.036 & 143.6 \\
\hline 21 & 0.03 & 177.85 & 0.03 & 187.61 & 0.036 & 144.4 \\
\hline 22 & 0.045 & 177.9 & 0.045 & 187.64 & 0.027 & 145 \\
\hline 23 & 0.012 & 179.5 & 0.012 & 189.5 & 0.036 & 145.1 \\
\hline 24 & 0.018 & 181.75 & 0.018 & 192.05 & 0.027 & 146.5 \\
\hline 25 & 0.045 & 183.1 & 0.045 & 193.56 & 0.036 & 147.9 \\
\hline 26 & 0.018 & 184.75 & 0.018 & 195.45 & 0.027 & 148.5 \\
\hline 27 & 0.027 & 187 & 0.027 & 198 & 0.027 & 150 \\
\hline
\end{tabular}


Table 2b. Lotteries Employed in the Experiment (continued)

\begin{tabular}{|c|c|c|c|c|c|c|}
\hline & $\mathrm{S} 1 \mathrm{~s}$ & & $\mathrm{~S} 2 \mathrm{~s}$ & & $\mathrm{~s} 3 \mathrm{~s}$ & \\
\hline Outcome & Probability & Return & Probability & Return & Probability & Return \\
\hline 1 & 0.5 & 75 & 0.5 & 68 & 0.4 & 95 \\
\hline 2 & 0.2 & 112 & 0.2 & 113 & 0.3 & 100 \\
\hline \multirow[t]{2}{*}{3} & 0.3 & 157 & 0.3 & 168 & 0.3 & 130 \\
\hline & $\mathrm{C}_{\mathrm{s}}$ & & $\mathrm{C} 2 \mathrm{~s}$ & & $\mathrm{C} 3 \mathrm{~s}$ & \\
\hline Outcome & Probability & Return & Probability & Return & Probability & Return \\
\hline 1 & 0.25 & 75 & 0.25 & 68 & 0.16 & 95 \\
\hline 2 & 0.2 & 93.5 & 0.2 & 90.5 & 0.24 & 97.5 \\
\hline 3 & 0.04 & 112 & 0.04 & 113 & 0.09 & 100 \\
\hline 4 & 0.3 & 116 & 0.3 & 118 & 0.24 & 112.5 \\
\hline 5 & 0.12 & 134.5 & 0.12 & 140.5 & 0.18 & 115 \\
\hline \multirow[t]{2}{*}{6} & 0.09 & 157 & 0.09 & 168 & 0.09 & 130 \\
\hline & VC1s & & $\mathrm{VC} 2 \mathrm{~s}$ & & VC3s & \\
\hline Outcome & Probability & Return & Probability & Return & Probability & Return \\
\hline 1 & 0.125 & 75 & 0.125 & 68 & 0.064 & 95 \\
\hline 2 & 0.05 & 76.11 & 0.05 & 69.35 & 0.048 & 95.15 \\
\hline 3 & 0.075 & 77.46 & 0.075 & 71 & 0.048 & 95.35 \\
\hline 4 & 0.05 & 77.59 & 0.05 & 71.15 & 0.036 & 95.5 \\
\hline 5 & 0.02 & 78.7 & 0.02 & 72.5 & 0.048 & 96.05 \\
\hline 6 & 0.03 & 80.05 & 0.03 & 74.15 & 0.036 & 96.4 \\
\hline 7 & 0.075 & 80.74 & 0.075 & 75 & 0.048 & 97.45 \\
\hline 8 & 0.03 & 81.85 & 0.03 & 76.35 & 0.036 & 97.6 \\
\hline 9 & 0.045 & 83.2 & 0.045 & 78 & 0.036 & 98.5 \\
\hline 10 & 0.05 & 108.3 & 0.05 & 108.5 & 0.048 & 99.5 \\
\hline 11 & 0.02 & 109.41 & 0.02 & 109.85 & 0.036 & 99.65 \\
\hline 12 & 0.03 & 110.76 & 0.03 & 111.5 & 0.036 & 99.85 \\
\hline 13 & 0.02 & 110.89 & 0.02 & 111.65 & 0.027 & 100 \\
\hline 14 & 0.008 & 112 & 0.008 & 113 & 0.036 & 100.55 \\
\hline 15 & 0.012 & 113.35 & 0.012 & 114.65 & 0.027 & 100.9 \\
\hline 16 & 0.03 & 114.04 & 0.03 & 115.5 & 0.036 & 101.95 \\
\hline 17 & 0.012 & 115.15 & 0.012 & 116.85 & 0.027 & 102.1 \\
\hline 18 & 0.018 & 116.5 & 0.018 & 118.5 & 0.027 & 103 \\
\hline 19 & 0.075 & 148.8 & 0.075 & 158 & 0.048 & 126.5 \\
\hline 20 & 0.03 & 149.91 & 0.03 & 159.35 & 0.036 & 126.65 \\
\hline 21 & 0.045 & 151.26 & 0.045 & 161 & 0.036 & 126.85 \\
\hline 22 & 0.03 & 151.39 & 0.03 & 161.15 & 0.027 & 127 \\
\hline 23 & 0.012 & 152.5 & 0.012 & 162.5 & 0.036 & 127.55 \\
\hline 24 & 0.018 & 153.85 & 0.018 & 164.15 & 0.027 & 127.9 \\
\hline 25 & 0.045 & 154.54 & 0.045 & 165 & 0.036 & 128.95 \\
\hline 26 & 0.018 & 155.65 & 0.018 & 166.35 & 0.027 & 129.1 \\
\hline 27 & 0.027 & 157 & 0.027 & 168 & 0.027 & 130 \\
\hline
\end{tabular}


Table 3 - Demographic Composition of the Sample

\begin{tabular}{lllllll}
\hline \hline Gender & \multicolumn{6}{l}{ Country } \\
\cline { 2 - 7 } & UK & China & Hong Kong & Other & Unknown & Sample \\
Female & $40 \%$ & $83 \%$ & $50 \%$ & $72 \%$ & $100 \%$ & $66 \%$ \\
Male & $60 \%$ & $17 \%$ & $50 \%$ & $28 \%$ & $0 \%$ & $34 \%$ \\
Sample & $25 \%$ & $37 \%$ & $10 \%$ & $25 \%$ & $3 \%$ & $100 \%$ \\
& & & & & & \\
\hline \hline & $\mathrm{N}$ & mean & s.d & $\min$ & $\max$ & \\
\hline \hline Age & 80 & 22.63 & 2.88 & 18 & 34 & \\
\hline \hline
\end{tabular}


Table 4 - Proportions of Safe Choices

\begin{tabular}{cccc}
\hline \hline $\begin{array}{c}\text { Problem } \\
\text { type }\end{array}$ & Lotteries & Number of decisions & Proportion of Safe choices \\
\hline 1 & SW-S & 440 & 0.78 \\
2 & SW-C & 480 & 0.73 \\
3 & SW-VC & 480 & 0.76 \\
4 & C-S & 480 & 0.65 \\
5 & VC-S & 440 & 0.58 \\
6 & C-VC & 480 & 0.52 \\
7 & Ss-S & 480 & 0.69 \\
8 & Cs-C & 480 & 0.62 \\
9 & VCs-VC & 480 & 0.70 \\
\hline \hline
\end{tabular}


Table 5: Reversal patterns for selected task-pairs, and McNemar test results for complexity aversion

\begin{tabular}{|l|l|c|c|c|c|c|c|c|c|}
\hline pair a & pair $b$ & $n$ & $R R$ & $R S$ & $S R$ & SS & McNemar & McNemar & Conclude \\
\hline & & & & & & & $\chi^{2}(1)$ & $p$-value & \\
\hline Phase 1 & & & & & & & & & \\
\hline SW-VC1 & VC1 -S1 & 80 & 4 & 7 & 22 & 47 & 7.75 & 0.005 & CA** $^{* * 1}$ \\
\hline SW-S1 & C1-S1 & 80 & 8 & 7 & 14 & 51 & 2.33 & 0.126 & \\
\hline SW-VC2 & VC2-S2 & 80 & 5 & 11 & 27 & 37 & 6.73 & 0.009 & CA** $^{* * 1}$ \\
\hline SW-S2 & C2-S2 & 80 & 10 & 6 & 14 & 50 & 3.2 & 0.073 & \\
\hline SW-VC3 & VC3-S3 & 40 & 4 & 7 & 17 & 12 & 4.17 & 0.041 & CA* $^{*}$ \\
\hline SW-S3 & C3-S3 & 40 & 7 & 5 & 17 & 11 & 6.55 & 0.011 & CA* $^{*}$ \\
\hline & & & & & & & & & \\
\hline Phase 2 & & & & & & & & & \\
\hline SW-VC1 & VC1-S1 & 80 & 5 & 11 & 28 & 36 & 7.41 & 0.006 & CA** $^{*}$ \\
\hline SW-S1 & C1-S1 & 80 & 6 & 9 & 15 & 50 & 1.5 & 0.220 & \\
\hline SW-VC2 & VC2-S2 & 80 & 5 & 15 & 26 & 34 & 2.95 & 0.085 & \\
\hline SW-S2 & C2-S2 & 80 & 9 & 11 & 19 & 41 & 2.13 & 0.144 & \\
\hline SW-VC3 & VC3-S3 & 80 & 13 & 10 & 31 & 26 & 10.75 & 0.001 & CA** $^{* * 1}$ \\
\hline SW-S3 & C3-S3 & 80 & 12 & 9 & 25 & 34 & 7.52 & 0.006 & CA** $^{* * 1}$ \\
\hline & & & & & & & & & \\
\hline
\end{tabular}

Notes: In final column, an empty cell indicates no evidence of complexity aversion or complexity lovingness; $\mathrm{CA}^{*}$ indicates evidence of complexity aversion; $\mathrm{CA}^{* *}$ indicates strong evidence. 
Table 6a. Maximum Likelihood Estimates of Finite Mixture Random Effects Models.

\begin{tabular}{|c|c|c|c|}
\hline & Model 1 & Model 2 & Model 3 \\
\hline$\theta_{10}$ risk av. & $2.964 * *(0.153)$ & $5.595 * *(0.243)$ & $3.930 * *(0.532)$ \\
\hline$\eta_{1}$ & & & $3.521 * *(0.349)$ \\
\hline$\theta_{20}$ complex. av. & $0.031 *(0.015)$ & $0.714^{* *}(0.076)$ & $0.095(0.077)$ \\
\hline \multicolumn{4}{|l|}{$\theta_{21}$ experience } \\
\hline \multicolumn{4}{|l|}{$\theta_{22}$ male } \\
\hline \multicolumn{4}{|l|}{$\theta_{23}$ (age-18) } \\
\hline \multicolumn{4}{|l|}{$\theta_{24}$ non-UK } \\
\hline$\eta_{2}$ & & & $0.509 * *(0.065)$ \\
\hline$\rho$ & & & $-0.333^{* *}(0.127)$ \\
\hline \multicolumn{4}{|l|}{$\varphi$} \\
\hline \multicolumn{4}{|l|}{$\delta$} \\
\hline$\pi_{1} \mathrm{RN}+\mathrm{CN}$ & & $0.289 * *(0.050)$ & $0.000(0.060)$ \\
\hline$\pi_{2}$ CN only & & $0.554 * *(0.060)$ & $0.496 * *(0.120)$ \\
\hline$\pi_{3}$ RN only & & $0.088 * *(0.030)$ & $0.026(0.070)$ \\
\hline$\pi_{4}$ neither & 1 & $0.069 *(0.030)$ & $0.479 * *(0.120)$ \\
\hline$n$ & 80 & 80 & 80 \\
\hline$T$ (mean of) & 53 & 53 & 53 \\
\hline$k$ & 2 & 5 & 8 \\
\hline $\log L$ & -2668.98 & -2465.09 & -2429.03 \\
\hline$A / C=-2(\log L-k)$ & 5341.96 & 4940.18 & 4874.06 \\
\hline
\end{tabular}

Notes: Asymptotic standard errors in parentheses. ${ }^{*}$ indicates significance $(p<0.05) ;{ }^{* *}$ indicates strong significance $(\mathrm{p}<0.01)$. Model 1 (homogeneous) assumes all subjects have same RA and CA. Model 2 (finite mixture) assumes four different types; Model 3 (finite mixture random effects) assumes 4 types and continuous variation in both RA and CA. Asymptotic s.e.s in parentheses. $k$ is the number of (estimated) parameters. AIC is Akaike's Information Criterion (preferred model has lowest AIC). 
Table 6b. Maximum Likelihood Estimates of Finite Mixture Random Effects Models (continued).

\begin{tabular}{|c|c|c|c|}
\hline & Model 4 & Model 5 & Model 6 \\
\hline$\theta_{10}$ risk av. & $3.138 * *(0.406)$ & $3.289 * *(0.396)$ & $2.388 * *(0.320)$ \\
\hline$\eta_{1}$ & $2.495 * *(0.336)$ & $2.871^{* *}(0.303)$ & $2.174 * *(0.252)$ \\
\hline$\theta_{20}$ complex. av. & $0.224 * *(0.069)$ & $0.003(0.088)$ & $0.104(0.090)$ \\
\hline$\theta_{21}$ experience & $-0.006 * *(0001)$ & $-0.006 * *(0.001)$ & $-0.007^{* *}(0.001)$ \\
\hline$\theta_{22}$ male & & $-0.096(0.081)$ & $-0.096(0.102)$ \\
\hline$\theta_{23}$ (age-18) & & $0.029 *(0.014)$ & $0.037 *(0.017)$ \\
\hline$\theta_{24}$ non-UK & & $0.154 *(0.087)$ & $0.134(0.107)$ \\
\hline$\eta_{2}$ & $0.349 * *(0.052)$ & $0.291^{* *}(0.042)$ & $0.321 * *(0.051)$ \\
\hline$\rho$ & $-0.112(0.195)$ & $-0.143(0.107)$ & $0.128(0.092)$ \\
\hline$\varphi$ & $-0.008 * *(0.002)$ & $-0.008 * *(0.002)$ & $-0.009 * *(0.003)$ \\
\hline$\delta$ & & & $0.020 * *(0.003)$ \\
\hline$\pi_{1} \mathrm{RN}+\mathrm{CN}$ & & & \\
\hline$\pi_{2}$ CN only & $0.356 * *(0.118)$ & $0.323^{* *}(0.120)$ & $0.325^{* *}(0.100)$ \\
\hline$\pi_{3}$ RN only & & & \\
\hline$\pi_{4}$ neither & $0.644 * *(0.118)$ & $0.677^{* *}(0.120)$ & $0.675^{* * *}(0.100)$ \\
\hline$n$ & 80 & 80 & 80 \\
\hline$T$ (mean of) & 53 & 53 & 53 \\
\hline$k$ & 8 & 11 & 12 \\
\hline $\log L$ & -2418.39 & -2410.45 & -2362.35 \\
\hline$A I C=-2(\log L-k)$ & 4852.78 & 4842.90 & 4748.7 \\
\hline
\end{tabular}

Notes: Asymptotic standard errors in parentheses. ${ }^{*}$ indicates significance $(p<0.05) ;{ }^{* *}$ indicates strong significance $(p<0.01)$. Model 4 (finite mixture random effects with experience) assumes 2 types, and allows for effect of experience in CA and error variance. Model 5 (finite mixture random effects with experience and subject characteristics) allows CA to depend on subject characteristics as well as experience. Model 6 assumes Prospective Reference Theory in place of EUT. Asymptotic s.e.s in parentheses. $k$ is the number of (estimated) parameters. AIC is Akaike's Information Criterion (preferred model has lowest AIC). 
Table 7. Preferences Distribution Classification of the 80 Subjects

\begin{tabular}{|l|c|c|c|}
\hline & Complexity-loving & Complexity-neutral & Complexity-averse \\
\hline Risk-loving & 3 & 1 & 6 \\
\hline Risk-averse & 11 & 25 & 34 \\
\hline
\end{tabular}

Notes: each cell contains the number of subjects falling into each category on the basis of their posterior estimates of RA and CA obtained following estimation of model 6 in Table $6 \mathrm{~b}$.

Figure 1 - Proportions of Safe Choices

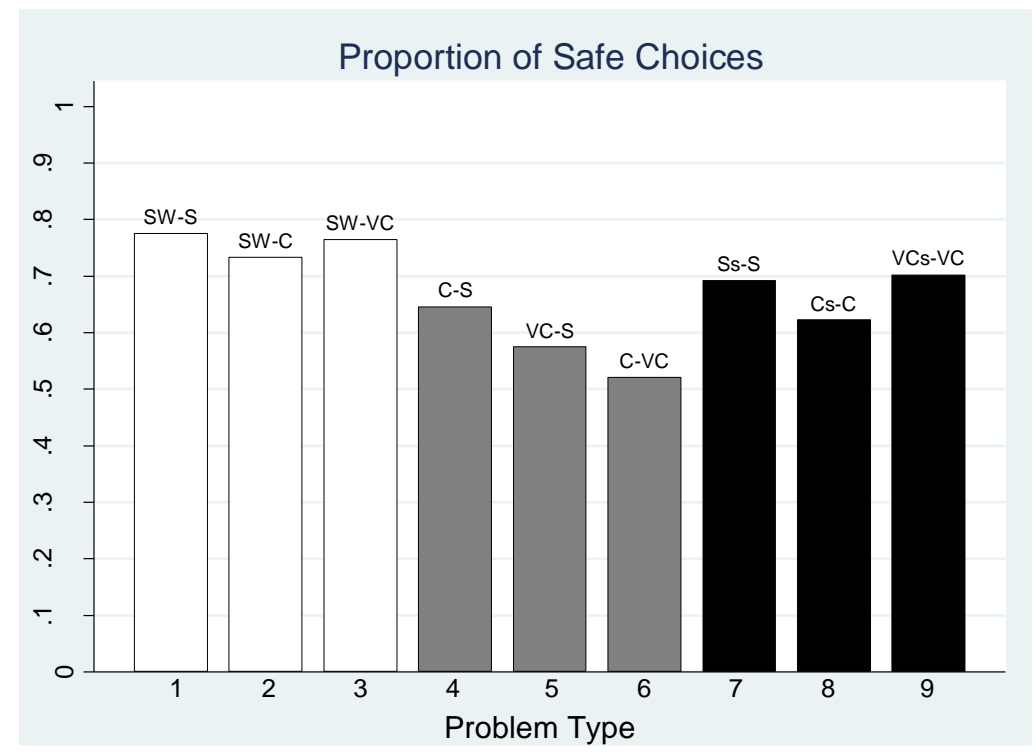


Figure 2: A frequency histogram of the 80 subjects' posterior probabilities of being type 2 (complexity-neutral) based on Model 6 in Table 6b

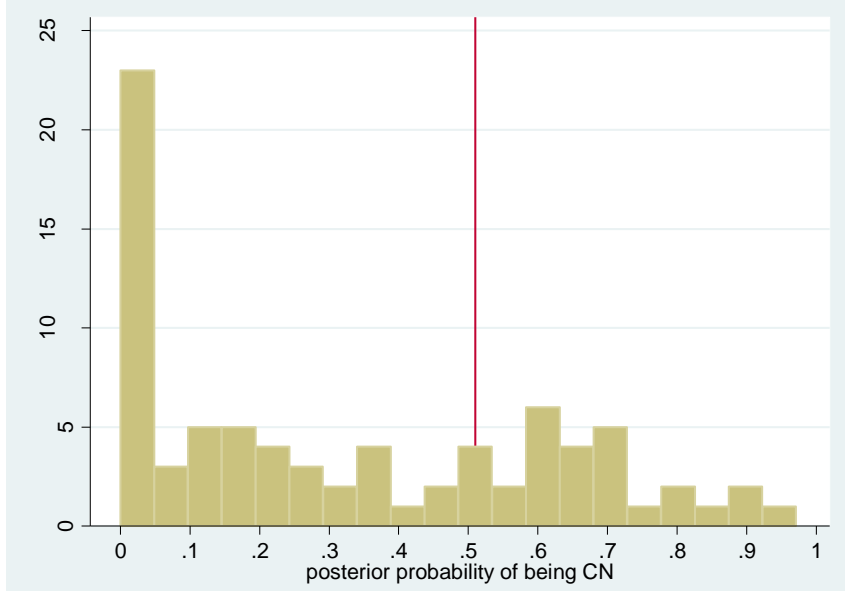

Notes: A vertical line is drawn at $0.51 .33 \%$ of the sample (26 subjects) are to the right of this, and on this basis they are classified as complexity neutral.

Figure 3: Posterior Estimates of $\alpha$ and $\gamma$ based on Model 6 in Table 6b

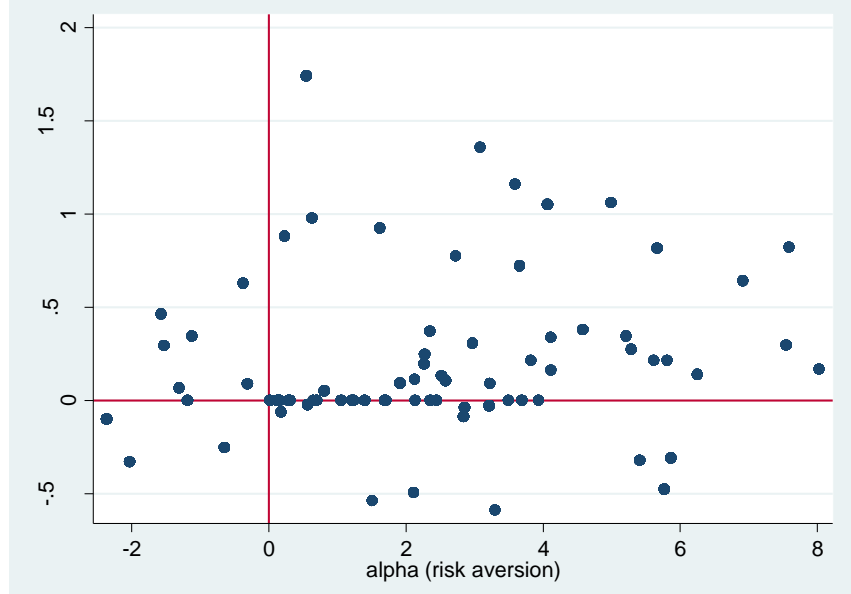

Notes: Observations on the horizontal axis are subjects whose posterior probabilities indicate that they are complexity-neutral. 